\title{
Force Spectroscopy with Quantitative On-Cantilever Force Control ${ }^{+}$
}

\author{
Wojciech Majstrzyk ${ }^{1, *}$, Karolina Orlowska ${ }^{1}$, Andrzej Sierakowski ${ }^{2}$, Rafał Dobrowolski ${ }^{2}$ and \\ Teodor Gotszalk ${ }^{1}$ \\ 1 Faculty of Microsystem Electronics and Photonics, Wroclaw University of Technology, 50-372 Wrocław, \\ Poland; karolina.orlowska@pwr.edu.pl (K.O.); teodor.gotszalk@pwr.edu.pl (T.G.) \\ 2 Division of Silicon Microsystem and Nanostructure Technology, Institute of Electron Technology, \\ 02-668 Warsaw, Poland; asierak@ite.wav.pl (A.S.); dobrowol3@tlen.pl (R.D.) \\ * Correspondence: wojciech.majstrzyk@pwr.edu.pl; Tel.: +48-71-320-30-38 \\ + Presented at the Eurosensors 2018 Conference, Graz, Austria, 9-12 September 2018.
}

Published: 23 November 2018

\begin{abstract}
In this work we propose a method for on-cantilever force control for application in force distance (F-z) spectroscopy for intermolecular interaction sensing. In this method we perform the F-z measurements with cantilevers integrating functionalized spheres attached to them. The F-z curve is obtained by controlling the Lorentz force acting at the cantilever free end. The intermolecular interaction forces are measured by measuring the force necessary to retract the cantilever from the surface. The required force is generated by the current passing through the cantilever. In this scenario we monitor the bending of cantilevers using optical beam deflection (OBD) method. Moreover, as deflection of the cantilever was electromagnetically controlled it was possible to calibrate the OBD response as well.
\end{abstract}

Keywords: Lorentz force; electromagnetic actuation; adhesion forces; cantilever sensor; active cantilever

\section{Introduction}

Since their first application in topography measurements in the Atomic Force Microscopy (AFM), silicon cantilevers have evolved into a new class of devices applied in metrology. The further development and their popularity has created a new field of scanning probe microscopy (SPM) technology. Only due to the modification of the cantilever properties, the SPM methods enable investigations of not only the surface topography but many other surface properties [1] like potential, magnetization, temperature, local force interactions [2]. The cantilevers, as micromechanical devices, are the versatile sensors with a wide field of applications in physical, chemical and biological sensing. In micro- and nanoelectromechanical systems (MEMS, NEMS) the movement of the device mechanical parts is controlled electrically. In this way, it is possible to excite static and resonance structure deflection. In case of the cantilevers, various methods of vibration excitation have been proposed including piezoelectric shakers, piezoelectric films, electrostatic, thermal and electromagnetic actuators.

In this article we present a method for F-z spectroscopy investigations enabling quantitative measurements of intermolecular bond forces based on an application of electromagnetically actuated array cantilevers. The electromagnetic cantilevers are based on silicon on insulator (SOI) technology, which was proposed in order to enable fabrication of micromechanical structures of defined parameters and allow investigations with a precise forcecontrol. 


\section{Materials and Methods}

In our experiments we use the electromagnetically actuated array cantilevers-Figure 1, fabricated in Institute of Electron Technology in Warsaw. The cantilevers have length ranging from 300 to $600 \mu \mathrm{m}, 100 \mu \mathrm{m}$ width and thickness of 1 or $1.5 \mu \mathrm{m}$. They are entirely made from Boron-doped silicon (BDS) basing on SOI technology.
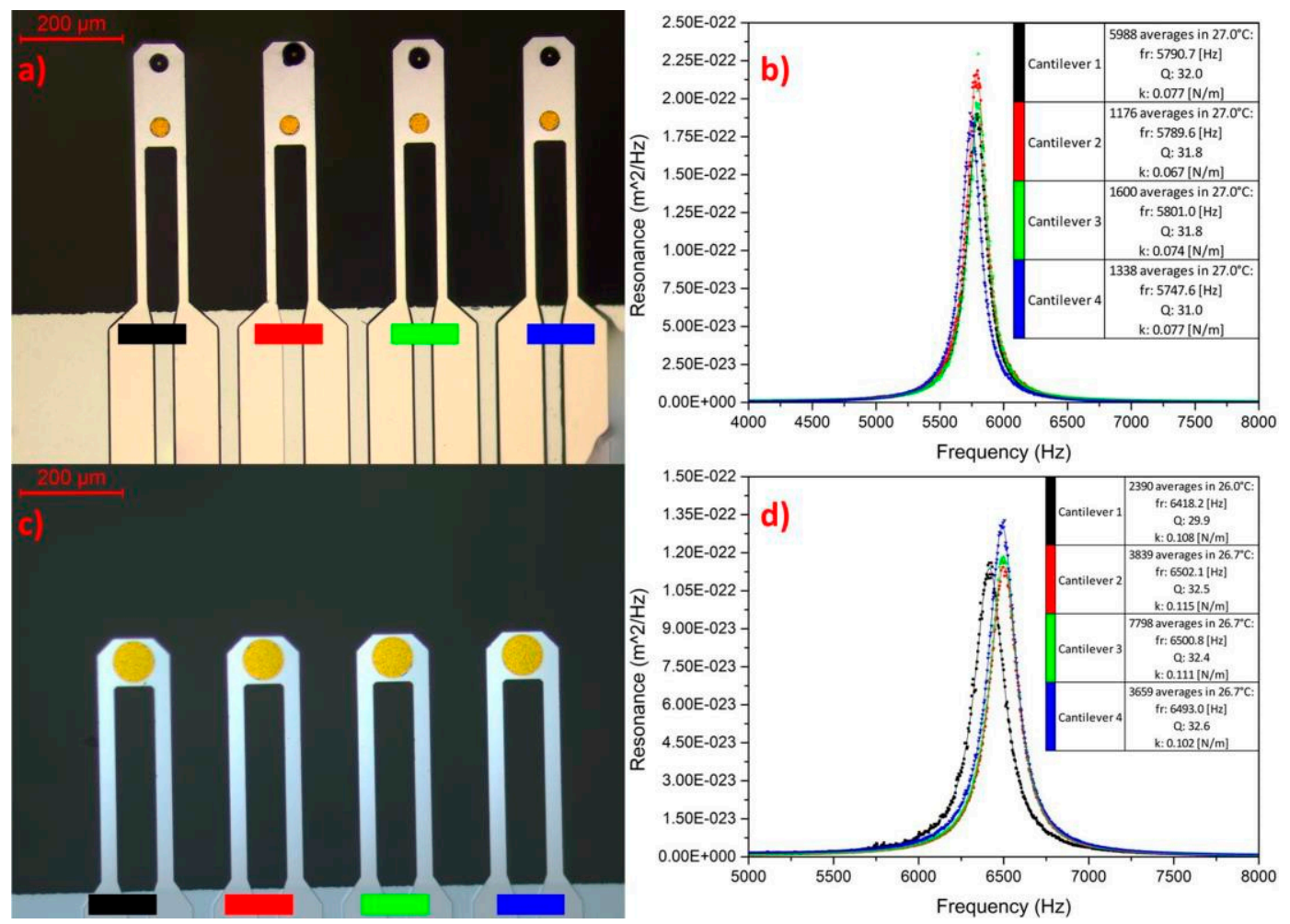

Figure 1. (a) Electromagnetically actuated array cantilevers with sphere mounted near their free end. Each cantilever integrates its own conductive loop, allowing for independent motion control; (b) thermomechanical noise measurements of the cantilevers (before sphere placement). The thermomechanical noise provides the estimated mechanical properties of each cantilever (c) Array with flat, gold functionalization layer. (d) Result of the thermomechanical noise measurements of the cantilevers with gold functionalization pads.

The boron doping allows for the current passing and Lorentz force actuation (1). In this technology the external magnetic field interacts with the current flowing through conductive loop producing the Lorentz force $\mathrm{F}$ [3-5]:

$$
\vec{F}=L_{L}(\vec{I} \times \vec{B})
$$

where $L_{L}$-distance between cantilever legs, $I$-current vector, $B$ - magnetic field vector. Our investigations follow two scenarios-Figure 2. We load the cantilevers with 32 to $40 \mu \mathrm{m}$ hollow glass microspheres made out of $\mathrm{SiO}_{2}$ and $\mathrm{SiO}_{2}$ coated with $50 \mathrm{~nm} \mathrm{Au}$-Figure 1a. In the second scenario we place the spheres onto prepared substrate with inverted pyramid arrays and perform investigations with cantilevers with flat gold area. The use of the microspheres allows for good contact region definition and allows us to apply different functional layers within single array [4]. The mass of the spheres is of ca. $20 \mathrm{ng}$ and does not significantly affect the control of the cantilevers movement.

The bending is observed using a typical optical beam deflection OBD detector, commonly used in AFM technology. The cantilever motion can be read from the OBD signal as well as from the current driving the cantilever [5]. In our method, the OBD acts as snap-in and pull-off interactions indicator. 


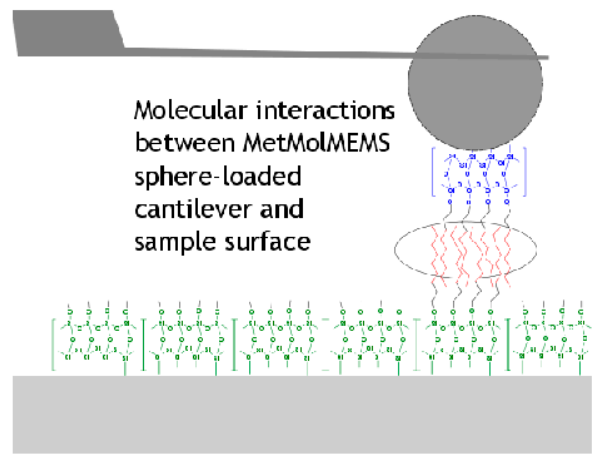

(a)

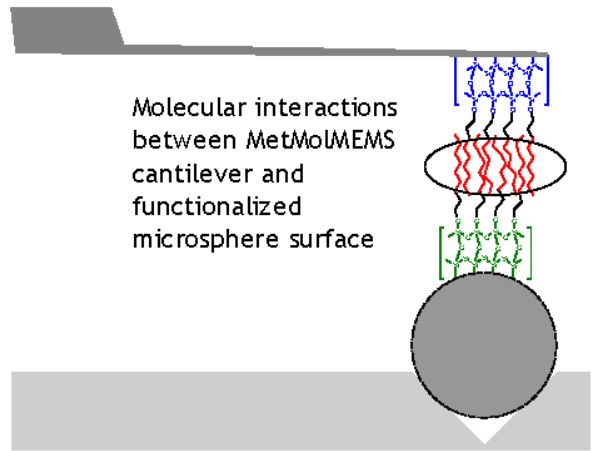

(b)

Figure 2. Schematic illustration of F-z spectroscopy investigation with $\mathrm{SiO}_{2}$ or Au coated $\mathrm{SiO}_{2}$ spheres; (a) This setup involves loading the cantilever with the sphere and investigating interactions between this functionalized sphere and flat surface; (b) setup, which involves sphere placed in with inverted pyramid arrays and investigating interactions between functionalized surfaces of sphere and flat gold layer on the cantilever.

\section{Results}

Figure 3 shows the results of the reverse F-z spectroscopy (RFS) measurement at ambient conditions. The use of this mode does not prohibit the use of classic Force Spectroscopy. The typical curve differs from one obtained in standard F-z mode. In standard mode the cantilever remains in constant position during the approach, but the sample moves towards it. When cantilever comes into contact it is bend. The deflection is matching the movement of the sample surface and the force acting on the cantilever tip is calculated from Hooke'slaw.

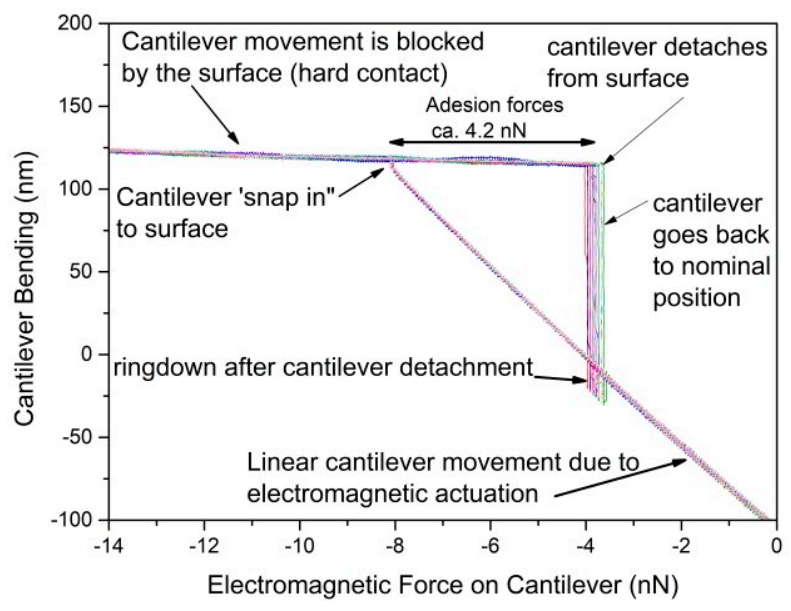

Figure 3. Result of the reverse F-z measurement. The cantilever approach and retracement is realized by the modulating current in Lorentz loop. This setup does not require Z-axis piezoelectric scanner.

In the RFS, the linear region of the OBD response is observed when the cantilever is not in contact with the investigated surface. When in contact, the position remains almost unchanged as additional force is insufficient to cause cantilever penetration into surface. However, with the increased force we can also perform indentation investigations on the sample. or better.

The electromagnetic driving gives us ability to linearly control the force with $20 \mathrm{pN}$ uncertainty

\section{Conclusions}

We have demonstrated the electromagnetically actuated array cantilevers with the ability to operate in F-z spectroscopy. The proposed cantilever utilizes electromagnetic actuation to perform 
so-called RFS investigations. Due to the precise control of the cantilever motion, we obtain quantitative information about adhesion forces between cantilever and sample.

Acknowledgments: The fabrication of cantilevers and measurements of the electromagnetic actuation were done within the NCN OPUS 9 Grant- "Metrology of molecular interactions using electromagnetically actuated MEMS force sensors-MetMolMEMS" (Grant No. 2015/17/B/ST7/03876).

Conflicts of Interest: The authors declare no conflict of interest.

\section{References}

1. Lang, H.P.; Hegner, M.; Meyer, E.; Gerber, C. Nanomechanics from atomic resolution to molecular recognition based on atomic force microscopy technology. Nanotechnology 2002, 13, R29-R36, doi:10.1088/0957-4484/13/5/20.

2. Moy, V.T.; Florin, E.L.; Gaub, H.E. Intermolecular forces and energies between ligands and receptors. Science 1994, 266, 257-259, doi:10.1126/science.7939660.

3. Buguin, A.; Du Roure, O.; Silberzan, P. Active atomic force microscopy cantilevers for imaging in liquids. Appl. Phy. Lett. 2001, 78, 2982-2984 doi:10.1063/1.1371250.

4. Nieradka, K.; Kopiec, D.; Małozięć, G.; Kowalska, Z.; Grabiec, P.; Janus, P.; Sierakowski, A.; Domański, K.; Gotszalk, T. Fabrication and characterization of electromagnetically actuated microcantilevers for biochemical sensing, parallel AFM and nanomanipulation. Microelectron. Eng. 2012, 98, 676-679, doi:10.1016/j.mee.2012.06.019.

5. Kopiec, D.; Pałetko, P.; Nieradka, K.; Majstrzyk, W.; Kunicki, P.; Sierakowski, A.; Jóźwiak G.; Gotszalk, T. Closed-loop surface stress compensation with an electromagnetically actuated microcantilever. Sens. Actuat. B Chem. 2015, 213, 566-573, doi:10.1016/j.snb.2015.03.001.

(C) 2018 by the authors. Licensee MDPI, Basel, Switzerland. This article is an open access article distributed under the terms and conditions of the Creative Commons Attribution (CC BY) license (http://creativecommons.org/licenses/by/4.0/). 